Blasco, M.J.; Casals, M.; Fernández-Villa, T.; Molina, A.J.; Martínez, F.V.; Langohr, K.; Ayán, C.; Martín, A. y Martín, V. (2018) Lesiones en la lucha tradicional: el caso de la lucha leonesa (20052015) / Injuries in Traditional Wrestling: The Leonese Wrestling Case Study (2005-2015). Revista Internacional de Medicina y Ciencias de la Actividad Física y el Deporte vol. 18 (72) pp. 723-736 Http://cdeporte.rediris.es/revista/revista72/artlesiones968.htm

DOI: http://doi.org/10.15366/rimcafd2018.72.008

\title{
ORIGINAL
}

\section{LESIONES EN LA LUCHA TRADICIONAL: EL CASO DE LA LUCHA LEONESA (2005-2015)}

\section{INJURIES IN TRADITIONAL WRESTLING: THE LEONESE WRESTLING CASE STUDY (2005-2015)}

\author{
Blasco, M.J.1; Casals, M.2; Fernández-Villa, T.3; Molina, A.J.4; Martínez, \\ F.V. ${ }^{5}$; Langohr, K. ${ }^{6}$; Ayán, C. ${ }^{7}$; Martín, A. ${ }^{8}$ y Martín, V. ${ }^{9}$
}

1 Complejo Asistencial Universitario de León. Sacyl. León (España) mblascocarmona@gmail.com

2 Centro de Estudios en Deporte y Actividad Física (CEEAF), Sport Performance Analysis Research Group, Universitat de Vic - Universitat Central de Catalunya (UVic-UCC) Barcelona (España) marticasals@gmail.com

3 Área de Medicina Preventiva y Salud Pública. Departamento de Ciencias Biomédicas. Universidad de León (España). Grupo de Investigación en Interacciones Gen-Ambiente y Salud. Instituto de Biomedicina (IBIOMED). Universidad de León (España) tferv@unileon.es

4 Área de Medicina Preventiva y Salud Pública. Departamento de Ciencias Biomédicas. Universidad de León (España). Grupo de Investigación en Interacciones Gen-Ambiente y Salud. Instituto de Biomedicina (IBIOMED). Universidad de León (España) ajmolt@unileon.es

${ }^{5}$ Centro de Salud de Mansilla de las Mulas. Gerencia de Atención Primaria de León. Sacyl. León (España) fvmga17@gmail.com

${ }^{6}$ Departamento de Estadística e Investigación Operativa, Universitat Politècnica de Catalunya / BARCELONATECH, Barcelona (España) klaus.langohr@upc.edu

${ }^{7}$ Facultad de Educación y Ciencias del Deporte. Departamento de Didácticas Especiales. WellMove Research Group. Universidad de Vigo (España) cayan@uvigo.es

${ }^{8}$ Servicios Médicos. Burela Fútbol Sala. Burela. Lugo (España) artumagar@gmail.com

${ }^{9}$ Centro de Investigación Biomédica en Red de Epidemiología y Salud Pública (CIBERESP), Instituto de Salud Carlos III, Madrid (España). Área de Medicina Preventiva y Salud Pública. Departamento de Ciencias Biomédicas. Universidad de León (España). Grupo de Investigación en Interacciones Gen-Ambiente y Salud. Instituto de Biomedicina (IBIOMED). Universidad de León (España) vicente.martin@unileon.es

\section{AGRADECIMIENTOS}

Este trabajo contó con financiación parcial de la Diputación de León, la Federación Territorial de Castilla y León de Lucha y CIBERESP (MTM2015-64465-C2-1-R; MINECO / FEDER).

Código UNESCO / UNESCO code: 3212 Salud Pública / Public Health Clasificación Consejo de Europa / Council of Europe classification: 11 Medicina del deporte /Sports Medicine 
Recibido 10 de diciembre de 2016 Received December 10, 2016

Aceptado 30 de noviembre de 2017 Accepted November 30, 2017

\section{RESUMEN}

El objetivo de este estudio fue describir la localización anatómica, el tipo y la gravedad de las lesiones ocurridas en las competiciones oficiales de las ligas de verano masculinas (2005-2015) de Lucha Leonesa. Al objeto de realizar un análisis más profundo, se tuvo en cuenta, el perfil del luchador. Se produjeron un total de 401 lesiones, siendo los luchadores de mayor nivel los que menos número de lesiones sufrieron. Las contusiones fueron las lesiones más frecuentes $(41,4 \%)$ pero entre las graves fueron los esguinces $(42,3 \%)$. Los miembros inferiores fueron la localización más frecuente en el total de lesiones $(38,2 \%)$ y entre las graves $(45,4 \%)$. La rodilla, el tórax y el hombro acumulaban casi la mitad de las lesiones y el $68 \%$ de las lesiones graves. Los resultados obtenidos sugieren que las características de las lesiones que se pueden observar en la lucha leonesa son similares a las de otros deportes de combate.

PALABRAS CLAVE: lucha tradicional, lesiones, prevención de lesiones

\section{ABSTRACT}

This study aimed at describing the anatomical location, type and severity of the sport injuries observed during the Leonese Wrestling "masculine summer leagues" official tournaments (2005-2015). The wrestler's profile was taken into account as a way to achieve a deep analysis. A total of 401 injuries were registered. The best wrestlers were the ones that suffered the least number of injuries. Contusions were the type of injuries more frequently registered $(41.4 \%)$, while sprains were the most common cause of severe injury (42.3\%). The most frequent anatomical location affected and the one in which a great number of severe injuries occurred were the lower limbs (38.2\% and $45.4 \%)$. The knee, thorax, and shoulder accounted for almost half of the total injuries and represented $68 \%$ of the severe injuries. The obtained results suggest that Leonese Wrestling show a similar injury pattern to the one observed in other combat sports.

PALABRAS CLAVE: tradicional wrestling, injuries, injuries prevention

\section{INTRODUCCIÓN}

Todos los pueblos, en todas las épocas, han tenido alguna forma de lucha que arraigaba tanto en el ceremonial religioso y social como en la práctica deportiva. Actualmente, la lucha se conserva tanto asociada a la tradición como adaptada a las formas convencionales del deporte moderno, lo que la convierte probablemente en el deporte más antiguo (Khalili-Borna \& Honsik, 2005). Las Luchas Tradicionales reflejan una manera de vivir, de relacionarse, por lo que son consideradas como un patrimonio cultural inmaterial de la humanidad que 
debe de ser reconocido y protegido (United Nations Educational, Scientific and Cultural Organization [UNESCO], 2006).

La Lucha Leonesa (LL) o Aluche es una Lucha Tradicional, registrada y oficialmente reconocida por la Federación Internacional de Luchas Asociadas (FILA), la Asociación Europea de Lucha Tradicional (AELT) y la Asociación Internacional de Luchas al Cinto (IBWA) (Federación Territorial de Lucha Castila y León, 2007). Como deporte de combate que es, no está exenta de lesiones, muchas de ellas prevenibles (Martín, et al., 2013). Desde el punto de vista de la epidemiología lesional, la prevención se considera una de las estrategias primordiales a la hora de reducir el riesgo de lesión derivado de la práctica deportiva (Saragiotto, Di Pierro, \& Lopes, 2014). A este respecto, el recopilar información sobre las lesiones que ocurren en un deporte se considera un recurso primordial que permite el desarrollo de acciones preventivas (Dahlström, Jacobsson, \& Timpka, 2015), especialmente en los deportes de combate (Pocecco, et al., 2013). Por lo tanto, conocer la frecuencia, gravedad, el tipo y la localización de las lesiones que se producen en el Aluche va a ser de utilidad para diseñar estrategias de prevención y control, no solo para esta lucha, pues al compartir factores de riesgo y mecanismos lesionales con las demás Luchas Tradicionales, el conocimiento de lo que sucede en una puede ser extensivo a la mayoría de ellas. De esta manera, la disminución de la incidencia de lesiones podría producir un aumento en el número de practicantes y evitar la precoz retirada de algunos de ellos, lo que en conjunto podría facilitar la protección y la pervivencia de este variado patrimonio cultural de la humanidad y a su vez cumplir con el mandato del Comité Olímpico Internacional (COI), de prevenir lesiones con el fin de fomentar la práctica del deporte seguro (Ljungqvist, 2008).

Es por ello que el presente estudio se desarrolló con el objeto principal de aportar información sobre la localización anatómica, tipo, y gravedad de las lesiones que se producen en LL. Un objetivo secundario fue el de determinar si las características de las lesiones observadas en este deporte guardan similitud entre los diferentes tipos de luchadores.

\section{MATERIAL Y MÉTODOS}

\subsection{LUCHA LEONESA}

La LL es un deporte de combate en el que dos participantes con agarre fijo a sendos cinturones tratan de derribar a su contrario mediante una serie de mañas (técnicas de la LL). El agarre es característico de la LL, el pulgar de una mano se introduce entre el cinto y la espalda, bien de abajo arriba o bien de arriba abajo (Espartero \& Martín, 1995), los otros dedos van por fuera y cierran la mano; la mano contraria pasa por encima del brazo del oponente y le agarra el cinto por el otro costado, los cuatro dedos se introducen entre el cinturón y el vientre de abajo arriba y es el pulgar el que cierra la mano por fuera. Vence el primero que en un tiempo tasado de combate tiene mayor puntuación o el primero en sumar dos caídas o cuatro puntos. Según las caídas la forma de puntuar es: 
- Caída entera o su equivalencia a dos puntos: cuando el luchador que la sufra hiciera contacto en el suelo con su espalda, entendiendo por espalda desde la región glútea hasta la última vértebra cervical. También el apoyo en tierra del hombro por su parte posterior.

- Media caída o un punto: caída a tierra por parte de uno de los luchadores haciendo contacto en ésta con el vientre; al soltarse sin previo consentimiento del árbitro; tomar contacto con tierra en plena caída con la parte transversal del hombro, con el brazo o la mano; y todo contacto con la cabeza para evitar la caída o aprovechar dicho apoyo.

El combate tiene lugar en el corro, el cual se organiza tradicionalmente sobre césped o bien sobre colchoneta u otro sistema tal que, igual que los anteriores, impida que cualquier contacto violento del luchador contra el suelo sea peligroso. Las dimensiones del terreno serán las de un círculo de 18 metros de diámetro, como mínimo para los corros de categoría senior.

\subsection{LUCHADORES}

Se estudiaron luchadores senior (16 años en adelante).

\subsection{TIPO DE ESTUDIO}

Se realizó un estudio descriptivo observacional durante los corros oficiales de la liga de verano masculina celebrados entre 2005 y 2015.

\subsection{CRITERIO DE LESIÓN}

La lesión se definió como cualquier acción producida en un combate y que por herir al luchador, impide que el combate siga su curso o necesite asistencia sanitaria y que por prevención, impide hacer esfuerzos como entrenar o competir en otros combates y otras actividades durante al menos las veinticuatro horas siguientes (Jarret, Orwin, \& Dick, 1998).

\subsection{CLASIFICACIÓN DE LAS LESIONES}

Las lesiones se clasificaron de acuerdo a su localización anatómica, tipo y gravedad. A este respecto, se distinguió en función del número de días que el luchador estuvo inactivo como consecuencia de la lesión, entre: lesiones leves (de 1 a 7 días de inactividad), moderadas (entre 8 y 28 días de inactividad) y graves (más de 28 días de inactividad) (Hägglund, Walden, Bahr, \& Ekstrand, 2005). 


\subsection{PERFIL DEL LUCHADOR}

A lo largo del presente estudio se registró el número de corros a los que asistió cada luchador, la edad en la que se iniciaron en la LL, y puntuación obtenida en las competiciones. De este modo, se distinguieron los siguientes perfiles luchador: "Habitual" (asiste a más del 66\% de los corros celebrados) vs "No Habitual" (asiste a menos del 66\% de las competiciones); "Experimentado" (se inició en la LL antes de cumplir 14 años) vs "No Experimentado" (se inició en la LL con 14 años cumplidos) y "Ganador" (aquel luchador que tiene más caídas a favor que en contra) vs "No Ganador" (aquel que tiene más caídas en contras que a favor).

\subsection{RECOGIDA DE INFORMACIÓN}

La información se obtuvo mediante asistencia directa a las competiciones por el personal sanitario, los partes de asistencia a las mutuas de accidentes y mediante entrevistas personalizadas al final de cada temporada para localizar lesiones no recogidas o revisión de las mismas.

\subsection{CONFIDENCIALIDAD Y ASPECTOS ÉTICOS}

Se entregó una hoja de información y se obtuvo el consentimiento informado por escrito del deportista, siendo aprobado el estudio por el Comité de Ética de la Universidad de León. El estudio se realizó de acuerdo con las normas de la Declaración de Helsinki (World Medical Association, 2013) y siguiendo las directrices de la Comunidad Europea de Buenas Prácticas Clínicas (111/3976/88 de julio de 1990) y el marco jurídico español para la investigación clínica en Humanos (Real Decreto 561/1993 sobre ensayos clínicos).

\subsection{ANÁLISIS ESTADÍSTICOS}

Se llevó a cabo un análisis descriptivo de las lesiones calculando número y porcentaje de las mismas en función de la localización, el tipo de lesión y la gravedad, así como un análisis estratificado en función del perfil del luchador teniendo en cuenta la habitualidad en el deporte, la edad de inicio en la LL y la calidad del luchador.

\section{RESULTADOS}

Durante las competiciones oficiales en las ligas de verano entre los años 2005-2015 participaron 308 luchadores y se contabilizaron un total de 401 lesiones que fueron $24,2 \%$ graves, $29,2 \%$ moderadas y $46,6 \%$ leves (Tabla 1 ). Las lesiones más frecuentes fueron las contusiones $(41,4 \%)$ y los esguinces $(33,2 \%)$, si bien los esguinces fueron las lesiones más frecuentes entre las graves (42,3\%), mientras que las contusiones fueron las más frecuentes en las lesiones moderadas y leves (42,7\% y $52,9 \%$ respectivamente). 
Tabla 1. Tipos de lesiones según gravedad producidas en las competiciones oficiales de liga de verano de 2005-2015.

\begin{tabular}{lcccccccc}
\hline & \multicolumn{2}{c}{ Graves } & \multicolumn{2}{c}{ Moderadas } & \multicolumn{2}{c}{ Leves } & \multicolumn{2}{c}{ Total } \\
& $\mathrm{N}$ & $\%$ & $\mathrm{~N}$ & $\%$ & $\mathrm{~N}$ & $\%$ & $\mathrm{~N}$ & $\%$ \\
\hline Contusión & 17 & 17,5 & 50 & 42,7 & 99 & 52,9 & 166 & 41,4 \\
Dislocación & 15 & 15,5 & 1 & 0,9 & 3 & 1,6 & 19 & 4,7 \\
Distensión & 6 & 6,2 & 22 & 18,8 & 34 & 18,2 & 62 & 15,5 \\
Esguince & 41 & 42,3 & 42 & 35,9 & 50 & 26,7 & 133 & 33,2 \\
Fractura & 18 & 18,6 & 2 & 1,7 & 1 & 0,5 & 21 & 5,2 \\
Total & 97 & 24,2 & 117 & 29,2 & 187 & 46,6 & 401 & 100,0 \\
\hline
\end{tabular}

La tabla 2 muestra el análisis del tipo de lesiones producidas según gravedad, habitualidad, y perfil del luchador, no existiendo diferencias notorias en el patrón lesivo en ninguno de los análisis realizados. Según la habitualidad, se observó un mayor número de lesiones en aquellos luchadores que acudían con asiduidad a las competiciones (253 lesiones) con respecto a los que lo hacían de forma esporádica (148 lesiones), si bien el patrón lesivo se mantuvo en ambos casos, siendo las contusiones (40,7\% y 42,6\% respectivamente) y los esguinces $(34,4 \%$ y $31,1 \%$ respectivamente) los tipos de lesiones más frecuentes.

Respecto a la edad de inicio en la LL, aquellos luchadores que se iniciaron en el deporte con menos de 14 años tuvieron mayor número de lesiones (237 lesiones) que los que comenzaron a participar en las competiciones con 14 años o más (164 lesiones), siendo las contusiones (41,8\% y 40,9\% respectivamente) y los esguinces $(36,7 \%$ y $28,0 \%$ respectivamente) los tipos de lesiones más frecuentes.

En el análisis de calidad del luchador, aquellos cuyo perfil era ganador (con más caídas a favor que en contra) tuvieron menos lesiones (183) que los que se catalogaron bajo el perfil de no ganador o con más caídas en contra (218 lesiones), siendo el patrón lesivo similar a los análisis anteriores, destacando las contusiones y los esguinces como los tipos de lesiones más frecuentes. 
Tabla 2. Tipos de lesiones según gravedad, habitualidad, edad de inicio en la Lucha Leonesa y perfil del luchador, producidas en las competiciones oficiales de liga de verano de 2005 -2015.

\begin{tabular}{|c|c|c|c|c|c|c|c|c|}
\hline \multirow[t]{2}{*}{ Perfil del Luchador } & \multicolumn{2}{|c|}{ Graves } & \multicolumn{2}{|c|}{ Moderadas } & \multicolumn{2}{|c|}{ Leves } & \multicolumn{2}{|c|}{ Total } \\
\hline & $\mathrm{N}$ & $\%$ & $\mathrm{~N}$ & $\%$ & $\mathrm{~N}$ & $\%$ & $\mathrm{~N}$ & $\%$ \\
\hline \multicolumn{9}{|l|}{ Habitual } \\
\hline Contusión & 12 & 21,4 & 29 & 40,3 & 62 & 49,6 & 103 & 40,7 \\
\hline Dislocación & 11 & 19,6 & 1 & 1,4 & 3 & 2,4 & 15 & 5,9 \\
\hline Distensión & 4 & 7,1 & 10 & 13,9 & 23 & 18,4 & 37 & 14,6 \\
\hline Esguince & 20 & 35,7 & 31 & 43,1 & 36 & 28,8 & 87 & 34,4 \\
\hline Fractura & 9 & 16,1 & 1 & 1,4 & 1 & 0,8 & 11 & 4,3 \\
\hline Total & 56 & 22,1 & 72 & 28,5 & 125 & 49,4 & 253 & 100,0 \\
\hline \multicolumn{9}{|l|}{ No Habitual } \\
\hline Contusión & 5 & 12,2 & 21 & 46,7 & 37 & 59,7 & 63 & 42,6 \\
\hline Dislocación & 4 & 9,8 & 0 & 0,0 & 0 & 0,0 & 4 & 2,7 \\
\hline Distensión & 2 & 4,9 & 12 & 26,7 & 11 & 17,7 & 25 & 16,9 \\
\hline Esguince & 21 & 51,2 & 11 & 24,4 & 14 & 22,6 & 46 & 31,1 \\
\hline Fractura & 9 & 22,0 & 1 & 2,2 & 0 & 0,0 & 10 & 6,8 \\
\hline Total & 41 & 27,7 & 45 & 30,4 & 62 & 41,9 & 148 & 100,0 \\
\hline \multicolumn{9}{|l|}{ Experimentado } \\
\hline Contusión & 9 & 16,4 & 29 & 42,6 & 61 & 53,5 & 99 & 41,8 \\
\hline Dislocación & 8 & 14,5 & 1 & 1,5 & 1 & 0,9 & 10 & 4,2 \\
\hline Distensión & 3 & 5,5 & 11 & 16,2 & 18 & 15,8 & 32 & 13,5 \\
\hline Esguince & 28 & 50,9 & 26 & 38,2 & 33 & 28,9 & 87 & 36,7 \\
\hline Fractura & 7 & 12,7 & 1 & 1,5 & 1 & 0,9 & 9 & 3,8 \\
\hline Total & 55 & 23,2 & 68 & 28,7 & 114 & 48,1 & 237 & 100,0 \\
\hline \multicolumn{9}{|l|}{ No Experimentado } \\
\hline Contusión & 8 & 19,0 & 21 & 42,9 & 38 & 52,1 & 67 & 40,9 \\
\hline Dislocación & 7 & 16,7 & 0 & 0,0 & 2 & 2,7 & 9 & 5,5 \\
\hline Distensión & 3 & 7,1 & 11 & 22,4 & 16 & 21,9 & 30 & 18,3 \\
\hline Esguince & 13 & 31,0 & 16 & 32,7 & 17 & 23,3 & 46 & 28,0 \\
\hline Fractura & 11 & 26,2 & 1 & 2,0 & 0 & 0,0 & 12 & 7,3 \\
\hline Total & 42 & 25,6 & 49 & 29,9 & 73 & 44,5 & 164 & 100,0 \\
\hline \multicolumn{9}{|l|}{ Ganador } \\
\hline Contusión & 5 & 11,9 & 20 & 37,0 & 45 & 51,7 & 70 & 38,3 \\
\hline Dislocación & 7 & 16,7 & 1 & 1,9 & 1 & 1,1 & 9 & 4,9 \\
\hline Distensión & 3 & 7,1 & 9 & 16,7 & 16 & 18,4 & 28 & 15,3 \\
\hline Esguince & 22 & 52,4 & 23 & 42,6 & 24 & 27,6 & 69 & 37,7 \\
\hline Fractura & 5 & 11,9 & 1 & 1,9 & 1 & 1,1 & 7 & 3,8 \\
\hline Total & 42 & 23,0 & 54 & 29,5 & 87 & 47,5 & 183 & 100,0 \\
\hline \multicolumn{9}{|l|}{ No Ganador } \\
\hline Contusión & 12 & 21,8 & 30 & 47,6 & 54 & 54,0 & 96 & 44,0 \\
\hline Dislocación & 8 & 14,5 & 0 & 0,0 & 2 & 2,0 & 10 & 4,6 \\
\hline Distensión & 3 & 5,5 & 13 & 20,6 & 18 & 18,0 & 34 & 15,6 \\
\hline Esguince & 19 & 34,5 & 19 & 30,2 & 26 & 26,0 & 64 & 29,4 \\
\hline Fractura & 13 & 23,6 & 1 & 1,6 & 0 & 0,0 & 14 & 6,4 \\
\hline Total & 55 & 25,2 & 63 & 28,9 & 100 & 45,9 & 218 & 100,0 \\
\hline
\end{tabular}

Respecto a la localización, la Tabla 3 muestra la distribución de lesiones según zona anatómica y gravedad, observándose como las zonas más lesionadas fueron el miembro inferior $(38,2 \%)$, el miembro superior $(31,9 \%)$ y el 
abdomen o tórax (16,2\%); si bien en las lesiones graves esos porcentajes se incrementan, en el caso de los miembros inferiores, hasta el 45,4\%.

Tabla 3. Distribución de lesiones producidas en competiciones de ligas de verano de 20052015, según localización y gravedad.

\begin{tabular}{lcccccccc}
\hline & Graves & \multicolumn{3}{c}{ Moderadas } & \multicolumn{3}{c}{ Leves } & Total \\
& $\mathrm{N}$ & $\%$ & $\mathrm{~N}$ & $\%$ & $\mathrm{~N}$ & $\%$ & $\mathrm{~N}$ & $\%$ \\
\hline Cabeza, cara o cuello & 5 & 5,2 & 11 & 9,4 & 24 & 12,8 & 40 & 10,0 \\
Abdomen/Tórax & 12 & 12,4 & 30 & 25,6 & 23 & 12,3 & 65 & 16,2 \\
Espalda & 0 & 0,0 & 8 & 6,8 & 7 & 3,7 & 15 & 3,7 \\
Miembro superior & 36 & 37,1 & 31 & 26,5 & 61 & 32,6 & 128 & 31,9 \\
Miembro inferior & 44 & 45,4 & 37 & 31,6 & 72 & 38,5 & 153 & 38,2 \\
Total & 97 & 100,0 & 117 & 100,0 & 187 & 100,0 & 401 & 100,0 \\
\hline
\end{tabular}

De una forma más específica, se puede observar en la Tabla 4 y Tabla 5, como las lesiones de rodilla $(18,2 \%)$, tórax $(16,0 \%)$ y hombro $(13,5 \%)$ acumulan cerca de la mitad de las lesiones; y en el caso de las lesiones graves el 68,0\% de las lesiones, con el 34,0\%, $12,4 \%$ y $21,6 \%$ respectivamente. No observándose diferencias relevantes entre los diversos perfiles de luchador (Figura 1).

Tabla 4. Distribución de lesiones según zona anatómica y gravedad.

\begin{tabular}{lcccccccc}
\hline & Graves & \multicolumn{3}{c}{ Moderadas } & \multicolumn{3}{c}{ Leves } & Total \\
& $\mathrm{N}$ & $\%$ & $\mathrm{~N}$ & $\%$ & $\mathrm{~N}$ & $\%$ & $\mathrm{~N}$ & $\%$ \\
\hline Abdomen & 0 & 0.0 & 0 & 0.0 & 1 & 0.5 & 1 & 0.2 \\
Antebrazo & 3 & 3.1 & 1 & 0.9 & 6 & 3.2 & 10 & 2.5 \\
Brazo & 0 & 0.0 & 0 & 0.0 & 2 & 1.1 & 2 & 0.5 \\
Cabeza/Cara/Cuello & 5 & 5.2 & 11 & 9.4 & 24 & 12.8 & 40 & 10.0 \\
Cadera & 1 & 1.0 & 0 & 0.0 & 1 & 0.5 & 2 & 0.5 \\
Cara anterior del Tórax & 12 & 12.4 & 30 & 25.6 & 22 & 11.8 & 64 & 16.0 \\
Codo & 2 & 2.1 & 2 & 1.7 & 2 & 1.1 & 6 & 1.5 \\
Espalda & 0 & 0.0 & 8 & 6.8 & 7 & 3.7 & 15 & 3.7 \\
Hombro & 21 & 21.6 & 11 & 9.4 & 22 & 11.8 & 54 & 13.5 \\
Mano & 1 & 1.0 & 3 & 2.6 & 6 & 3.2 & 10 & 2.5 \\
Muñeca & 6 & 6.2 & 3 & 2.6 & 9 & 4.8 & 18 & 4.5 \\
Muslo & 3 & 3.1 & 12 & 10.3 & 14 & 7.5 & 29 & 7.2 \\
Otro dedo mano & 2 & 2.1 & 3 & 2.6 & 2 & 1.1 & 7 & 1.7 \\
Otro dedo pie & 1 & 1.0 & 2 & 1.7 & 7 & 3.7 & 10 & 2.5 \\
Pierna & 1 & 1.0 & 0 & 0.0 & 2 & 1.1 & 3 & 0.7 \\
Primer dedo mano & 1 & 1.0 & 8 & 6.8 & 12 & 6.4 & 21 & 5.2 \\
Primer dedo pie & 2 & 2.1 & 3 & 2.6 & 8 & 4.3 & 13 & 3.2 \\
Rodilla & 33 & 34.0 & 14 & 12.0 & 26 & 13.9 & 73 & 18.2 \\
Tobillo & 3 & 3.1 & 6 & 5.1 & 14 & 7.5 & 23 & 5.7 \\
Total & 97 & 24.2 & 117 & 29.2 & 187 & 46.6 & 401 & 100.0 \\
\hline
\end{tabular}


Tabla 5. Distribución de lesiones según localización, gravedad, habitualidad, edad de inicio y calidad del luchador.

\begin{tabular}{|c|c|c|c|c|c|c|c|c|}
\hline & \multicolumn{2}{|c|}{ Graves } & \multicolumn{2}{|c|}{ Moderadas } & \multicolumn{2}{|c|}{ Leves } & \multicolumn{2}{|c|}{ Total } \\
\hline & $\mathrm{N}$ & $\%$ & $\mathrm{~N}$ & $\%$ & $\mathrm{~N}$ & $\%$ & $\mathrm{~N}$ & $\%$ \\
\hline \multicolumn{9}{|l|}{ Habitual } \\
\hline Cabeza, cara o cuello & 2 & 3.6 & 7 & 9.7 & 15 & 12.0 & 24 & 9.5 \\
\hline Abdomen/Tórax & 8 & 14.3 & 22 & 30.6 & 13 & 10.4 & 43 & 17.0 \\
\hline Espalda & 0 & 0.0 & 2 & 2.8 & 3 & 2.4 & 5 & 2.0 \\
\hline Miembro superior & 21 & 37.5 & 18 & 25.0 & 44 & 35.2 & 83 & 32.8 \\
\hline Miembro inferior & 25 & 44.6 & 23 & 31.9 & 50 & 40.0 & 98 & 38.7 \\
\hline Total & 56 & 100.0 & 72 & 100.0 & 125 & 100.0 & 253 & 100.0 \\
\hline \multicolumn{9}{|l|}{ No habitual } \\
\hline Cabeza, cara o cuello & 3 & 7.3 & 4 & 8.9 & 9 & 14.5 & 16 & 10.8 \\
\hline Abdomen/Tórax & 4 & 9.8 & 8 & 17.8 & 10 & 16.1 & 22 & 14.9 \\
\hline Espalda & 0 & 0.0 & 6 & 13.3 & 4 & 6.5 & 10 & 6.8 \\
\hline Miembro superior & 15 & 36.6 & 13 & 28.9 & 17 & 27.4 & 45 & 30.4 \\
\hline Miembro inferior & 19 & 46.3 & 14 & 31.1 & 22 & 35.5 & 55 & 37.2 \\
\hline Total & 41 & 100.0 & 45 & 100.0 & 62 & 100.0 & 148 & 100.0 \\
\hline \multicolumn{9}{|l|}{ Experimentado } \\
\hline Cabeza, cara o cuello & 3 & 5.5 & 8 & 11.8 & 15 & 13.2 & 26 & 11.0 \\
\hline Abdomen/Tórax & 5 & 9.1 & 19 & 27.9 & 14 & 12.3 & 38 & 16.0 \\
\hline Espalda & 0 & 0.0 & 6 & 8.8 & 3 & 2.6 & 9 & 3.8 \\
\hline Miembro superior & 19 & 34.5 & 20 & 29.4 & 37 & 32.5 & 76 & 32.1 \\
\hline Miembro inferior & 28 & 50.9 & 15 & 22.1 & 45 & 39.5 & 88 & 37.1 \\
\hline Total & 55 & 100.0 & 68 & 100.0 & 114 & 100.0 & 237 & 100.0 \\
\hline \multicolumn{9}{|l|}{ No experimentado } \\
\hline Cabeza, cara o cuello & 2 & 4.8 & 3 & 6.1 & 9 & 12.3 & 14 & 8.5 \\
\hline Abdomen/Tórax & 7 & 16.7 & 11 & 22.4 & 9 & 12.3 & 27 & 16.5 \\
\hline Espalda & 0 & 0.0 & 2 & 4.1 & 4 & 5.5 & 6 & 3.7 \\
\hline Miembro superior & 17 & 40.5 & 11 & 22.4 & 24 & 32.9 & 52 & 31.7 \\
\hline Miembro inferior & 16 & 38.1 & 22 & 44.9 & 27 & 37.0 & 65 & 39.6 \\
\hline Total & 42 & 100.0 & 49 & 100.0 & 73 & 100.0 & 164 & 100.0 \\
\hline \multicolumn{9}{|l|}{ Perfil ganador } \\
\hline Cabeza, cara o cuello & 1 & 2.4 & 6 & 11.1 & 13 & 14.9 & 20 & 10.9 \\
\hline Abdomen/Tórax & 5 & 11.9 & 16 & 29.6 & 11 & 12.6 & 32 & 17.5 \\
\hline Espalda & 0 & 0.0 & 1 & 1.9 & 2 & 2.3 & 3 & 1.6 \\
\hline Miembro superior & 13 & 31.0 & 12 & 22.2 & 27 & 31.0 & 52 & 28.4 \\
\hline Miembro inferior & 23 & 54.8 & 19 & 35.2 & 34 & 39.1 & 76 & 41.5 \\
\hline Total & 42 & 100.0 & 54 & 100.0 & 87 & 100.0 & 183 & 100.0 \\
\hline \multicolumn{9}{|l|}{ Perfil no ganador } \\
\hline Cabeza, cara o cuello & 4 & 7.3 & 5 & 7.9 & 11 & 11.0 & 20 & 9.2 \\
\hline Abdomen/Tórax & 7 & 12.7 & 14 & 22.2 & 12 & 12.0 & 33 & 15.1 \\
\hline Espalda & 0 & 0.0 & 7 & 11.1 & 5 & 5.0 & 12 & 5.5 \\
\hline Miembro superior & 23 & 41.8 & 19 & 30.2 & 34 & 34.0 & 76 & 34.9 \\
\hline Miembro inferior & 21 & 38.2 & 18 & 28.6 & 38 & 38.0 & 77 & 35.3 \\
\hline Total & 55 & 100.0 & 63 & 100.0 & 100 & 100.0 & 218 & 100.0 \\
\hline
\end{tabular}




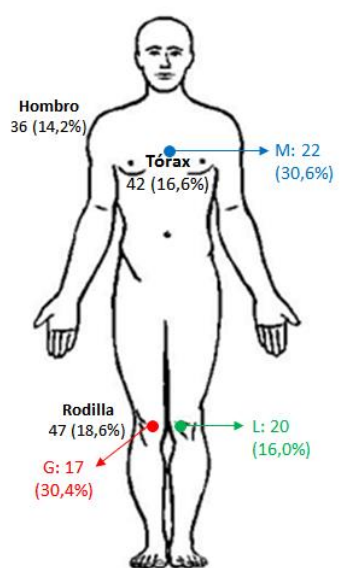

Habituales
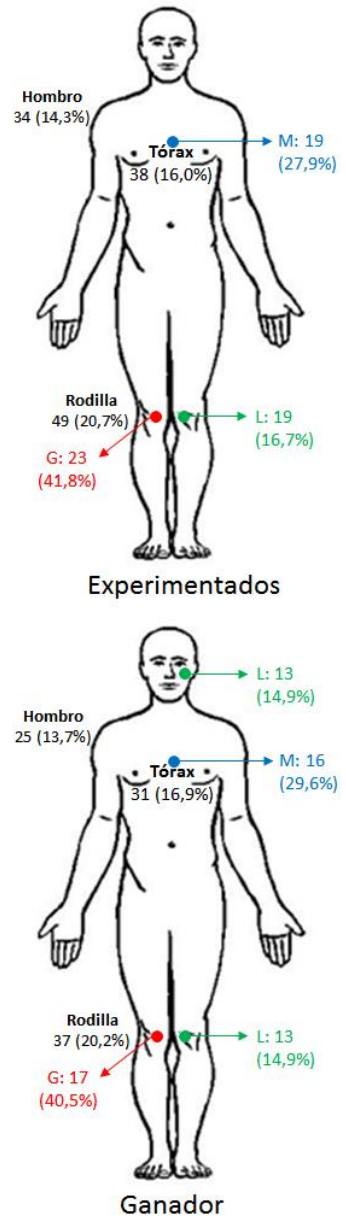

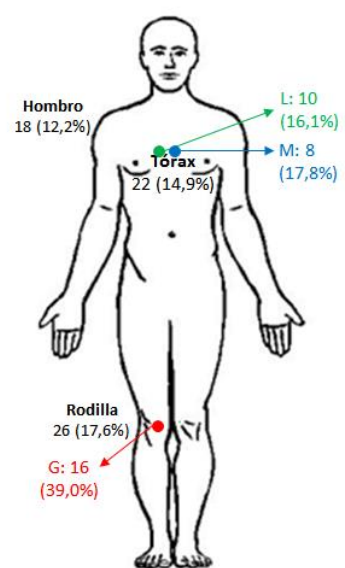

No habituales
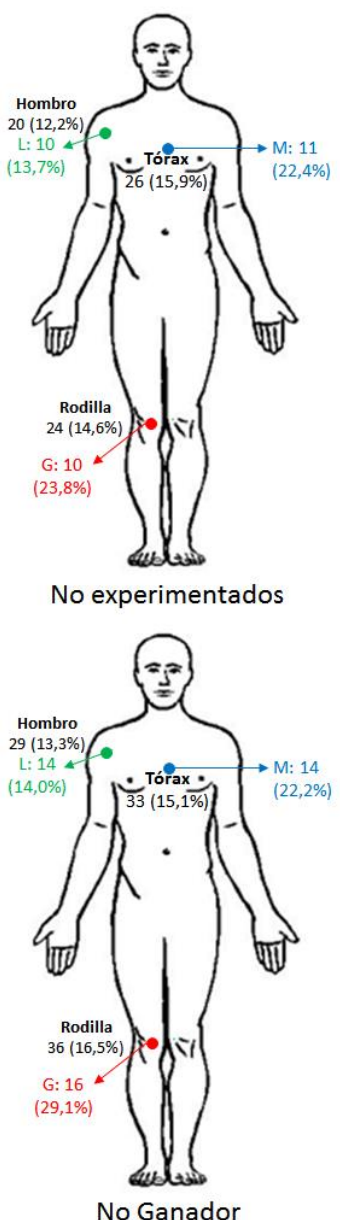

Figura 1. Distribución de las zonas anatómicas más lesionadas en general, según gravedad, habitualidad, y perfil del luchador. En negro aparecen las tres localizaciones más frecuentes de

las lesiones en general, mientras que en rojo, azul y verde se destaca la localización más frecuente en las lesiones graves, moderadas y leves respectivamente.

\section{DISCUSIÓN}

Este estudio tuvo como objetivo aportar información sobre la localización anatómica, el tipo y severidad de las lesiones que acontecen durante la práctica de la LL. Además, se tuvo en cuenta el perfil del luchador al objeto de realizar un análisis más completo. El proporcionar este tipo de información facilitará la puesta en marcha de 
mecanismos de prevención y control en las luchas tradicionales favoreciendo así la pervivencia de un patrimonio cultural único.

La lucha es un deporte de contacto que utiliza la fuerza, la resistencia y la velocidad, así como cualidades coordinativas de movilidad y de agilidad lo que de alguna manera va a condicionar el tipo de lesión. En el caso de la LL, el ser una lucha al cinto, el agarre también va a condicionar el tipo de lesión. (Martín, et al., 2013). Por todas estas razones, no es de extrañar que la rodilla, el hombro y el tórax fuesen las localizaciones anatómicas más afectadas y que las contusiones y los esguinces constituyesen las lesiones más frecuentes.

En lo que a la localización anatómica de las lesiones se refiere, entre la rodilla, el hombro y la cara anterior del tórax sumaron cerca de la mitad del total de las lesiones y dos terceras partes de las graves. El miembro inferior fue la zona corporal en la que se localizaron el mayor número de lesiones, siendo la rodilla la zona más afectada. Este hecho ha sido también observado en otra lucha tradicional española, la Lucha Canaria (Sous, et al., 2006), en la lucha libre (Jarret, Orwin, \& Dick, 1998) y en el judo (Green, Petrou, Fogarty-Hover, \& Rolf, 2007; Pierantozzi \& Muroni, 2009).

Un tercio de las lesiones se localizaron en la extremidad superior, tanto graves como en el cómputo total, hecho que también se ha constatado en los practicantes de lucha libre, (Caine, Young, \& Howe, 2009). En la LL se ha sugerido que la principal causa de las lesiones en la extremidad superior es la suelta (Ayán, et al., 2010). La suelta consiste en zafarse del agarre para obtener una menor penalización y se ha observado que aumenta el riesgo de lesiones, especialmente del miembro superior; de manera que eliminando las medias caídas puede reducir la incidencia de lesiones del miembro superior (Ayán, et al., 2010; Martín, et al. 2013). El hombro fuer la articulación del miembro superior que mayor número de lesiones sufrió, lo que está en línea con lo observado en deportes de combate similares, como el judo (Pocecco et al., 2013). Las lesiones más frecuentes obedecen a luxaciones de la articulación acromio-clavicular y en mayor medida gleno-humeral. Éstas últimas se producen al quedar el brazo fijado perpendicularmente al suelo o al adversario y describir el cuerpo un arco cuyo eje de giro que está situado en la articulación gleno-humeral.

Las lesiones en el tórax pueden ser consideradas como un aspecto peculiar de la LL. Las mismas, casi con exclusividad son debidas a contusiones costales consecuencia del empleo de un cinturón de piel sobre el que el contrincante ejerce una gran presión, a efectos de lograr un agarre sólido. Esto supone la creación de una mortaja sobre el tórax de manera que cualquier giro o golpe para a lesionar las costillas o los cartílagos costales.

El análisis pormenorizado de los datos registrados indicó que las contusiones y los esguinces supusieron cuatro de cada cinco lesiones y sólo en el caso de las lesiones graves no alcanzaron las dos terceras partes del total. Es esta una constante que se observa no solo en la lucha, dónde los golpes contra el suelo o el oponente y/o los giros bruscos, violentos, de las diferentes acciones técnicas son habituales, sino que también es el patrón que frecuentemente se puede encontrar en el judo y en otra lucha tradicional (Lucha Canaria) (Sous, et al., 2006). A este respecto, conviene remarcar que en ninguna otra disciplina se observa que el conjunto de estas lesiones alcance el $75 \%$ o el $66 \%$ del total de lesiones graves, como se ha observado en el presente estudio, pero si superan en todos los casos el 50\% de las lesiones (Green, Petrou, Fogarty-Hover, \& Rolf, 2007; Pocecco, et al., 2013). En otros deportes de combate como la lucha libre la lesión más habitual y que provoca un mayor tiempo de inactividad es la distensión muscular (Rechel, Yard, \& Comstock, 2008; Caine, Young, \& Howe, 2009). Sin embargo, en el 
presente trabajo, la distensión constituyó el tercer tipo de lesión más frecuente. Las diferencias en el agarre pueden explicar las discrepancias observadas entre ambas disciplinas.

Las luxaciones y las fracturas supusieron un porcentaje inferior al $10 \%$ en el total de las lesiones, si bien representaron un tercio de las lesiones graves. En ambos casos los mecanismos de producción son idénticos a los de las contusiones y/o esguinces pero sobrepasando el nivel de resistencia de la articulación o bien del hueso. En el judo, quizás el deporte de combate que más se asemeja a la LL, se han observado resultados similares a este respecto (Pocecco, et al., 2013).

Para establecer la gravedad de las lesiones se utilizó el criterio de "tiempo de inactividad", que si bien es cierto que en algunas ocasiones no se correlaciona con las consecuencias de las lesiones, es un criterio de utilidad, pues facilita la comparación entre diferentes disciplinas deportivas (Ekstrand, Gillquist, \& Liljedahl, 1983; Parkkari, et al., 2004). En nuestra serie, una de cada cuatro lesiones supuso más de 28 días de baja, lo que se encuentra próximo a los trabajos publicados sobre lucha libre dónde el $27 \%$ de las lesiones reportadas eran graves (Yard, Collins, Dick, \& Comstock, 2008) y un $32,6 \%$ de los practicante estuvieron más de 7 días de baja (Powell \& Barber-Foss, 1999a). En el caso del taekwondo se ha encontrado que el $26 \%$ de las lesiones supusieron entre 2-7 días de baja.

En otros deportes de combate el comportamiento de las lesiones ha sido distinto al aquí observado. Así, en deportistas olímpicos que participaron en combates de lucha libre y lucha grecorromana, se encontró que el $81,5 \%(22 / 27)$ de las lesiones fueron leves, el 18,5\% (5/27) fueron moderadas y no se observaron lesiones graves (Shadgan, Feldman, \& Jafari, 2010). Las diferencias observadas pueden ser explicadas en base a dos factores. El primero es el pequeño número de combates en el que se basó el estudio (328). El segundo, obedece a las peculiares características de la LL y de sus practicantes, que por lo general son amateurs que en muchas ocasiones compiten sin entrenamiento previo (actos lúdicos en las fiestas del pueblo). Además no hay controles médicos previos a la competición, el reglamento no prohíbe acciones y mañas de riesgo de lesión. Finalmente a esto se debe de añadir que luchadores presentan un bajo nivel de entrenamiento y participan en muchas competiciones muy concentradas llegando incluso a tomar parte en cinco y seis competiciones seguidas en cinco o seis días (Martín, et al., 2013). Por el contrario, los luchadores de categoría olímpica deben tomar parte en controles médicos muy rigurosos incluso antes de la competición y tienen una gran dedicación (cuasi-profesional); sus reglamentos son muy estrictos a la hora de evitar o prohibir acciones y maniobras de riesgo elevado de lesión y son luchadores de mucho tiempo dedicado al entrenamiento y de muy pocas pero intensas competiciones (Zetaruk, Violán, Zurakowski, \& Micheli, 2005). En el caso de la LL los luchadores son amateurs.

Como añadido al tradicional estudio descriptivo de lesiones deportivas, en esta investigación se optó por tratar de determinar si ciertas características de los luchadores lo predisponían más a sufrir lesiones más graves o de diferente localización o tipo. Los resultados obtenidos indicaron que el tipo, gravedad y zona anatómica afectada por las lesiones que ocurren en LL son similares entre los luchadores. Sin embargo, si se constató que los luchadores más experimentados y que participaban en un mayor número de competiciones, sufrían un mayor número de lesiones, lo que podría ser debido a que presentan un mayor tiempo de exposición a la práctica deportiva. En esta línea, se constató que los luchadores con un perfil ganador sufrían un menor número de lesiones. A este respecto, se podría hipotetizar que la condición física y habilidad técnica 
de los mismos les permitiese realizar las mañas con una mayor eficiencia biomecánica así como soportar mejor los lances potencialmente lesivos derivados del combate.

A pesar de la cuidadosa metodología empleada y el gran número de competiciones analizadas en este estudio existen varias limitaciones que deben ser reconocidas. En primer lugar, sólo se analizaron corros celebrados durante la liga de verano y luchadores senior. En segundo lugar, no se tuvo en cuenta un factor fundamental relacionado con en el perfil lesivo de las personas que practican deportes de lucha, como es la relación entre su peso y la categoría en la que principalmente compiten. Finalmente, es prudente reconocer que si bien la recogida de información fue exhaustiva, no se puede descartar que un número de lesiones leves no hayan sido detectadas o declaradas, al no darles relevancia los propios deportistas, y sería sensato pensar que el porcentaje de lesiones leves es mayor del reportado en este estudio.

\section{CONCLUSIÓN}

El tipo de lesión más frecuente en la LL es la contusión, siendo la rodilla, el hombro y el tórax, las zonas anatómicamente más afectadas. La mayor parte de las lesiones se concentran en los miembros inferiores, siendo una cuarta parte del total de las mismas graves. Este patrón lesional es similar al de otros deportes de combate. El tipo, localización y severidad de la lesión no presenta grandes diferencias entre los distintos perfiles de luchadores.

\section{REFERENCIAS BIBLIOGRÁFICAS}

Ayán, C., Molina, A., García, H., González, G., Álvarez, M., Férnandez, T., \& Martín, V. (2010). Efecto de una modificación reglamentaria en la incidencia de lesiones en lucha leonesa. Apunts. Medicina de l'Esport, 45(165), 17-22. https://doi.org/10.1016/j.apunts.2009.06.001

Caine, D., Young, K., \& Howe, W. (2009). Wrestling. In D. Caine, P. Harmer, \& M. Schiff (Eds.), Epidemiology of Injury in Olympic Sports (Vol. XVI). Oxford, UK: WileyBlackwell. https://doi.org/10.1002/9781444316872

Dahlström, Ö., Jacobsson, J., \& Timpka, T. (2015). Overcoming the organizationpractice barrier in sports injury prevention: A nonhierarchical organizational model. Scandinavian journal of medicine \& science in sports, 25(4). https://doi.org/10.1111/sms.12327

Ekstrand, J., Gillquist, J., \& Liljedahl, S. (1983). Prevention of soccer injuries. Supervision by doctor and physiotherapist. The American Jounal of Sport Medicine, 11(3), 116-120. https://doi.org/10.1177/036354658301100302

Espartero, J., \& Martín, J. (1995). Análisis conceptual, estructural formal y estructural dinámico de un deporte de lucha con agarre: la Lucha Leonesa. Perspectivas de la actividad física y el deporte, 17, $42-47$.

Federación Territorial de Lucha Castilla y León. (2007). Retrieved from http://www.luchaleonesa.es/fcllftp/estatutos_federacion_lucha_cyl_a.pdf

Green, C., Petrou, M., Fogarty-Hover, M., \& Rolf, C. (2007). Injuries among judokas during competition. Scandinavian Journal of Medicine \& Science in Sports, 17, 205-210.

Hägglund, M., Walden, M., Bahr, R., \& Ekstrand, J. (2005). Methods for epidemiological study of injuries to professional football players: developing the UEFA model. British Journal of Sport Medicine(39), 340-346. https://doi.org/10.1136/bjsm.2005.018267 
Jarret, G., Orwin, J., \& Dick, R. (1998). Injuries in collegiate wrestling. The American Journal of Sport Medicine, 26(5), 674-680. https://doi.org/10.1177/03635465980260051301

Khalili-Borna, D., \& Honsik, K. (2005). Wrestling and Sports Medicine. Current Sports Medicine Reports, 4(3), 144-149. https://doi.org/10.1097/01.CSMR.0000306197.51994.16

Ljungqvist, A. (2008). Sports Injury prevention: A key mandate for the IOC. British Journal of Sport Medicine, 42(6), 391.

Martín, V., Fernández, T., Ayán, C., Molina, A., García, H., Álvarez, M., \& Delgado, M. (2013). A success story: New rules and fewer injuries in traditional Leonese Wrestling (2006-12). Apunts. Medicina de l'Esport, 48(178), 55-61. https://doi.org/10.1016/i.apunts.2012.07.003

Parkkari, J., Kannus, P., Natri, A., Lapinleimu, I., Palvanen, M., Heiskanen, M., . . . Järvinen, M. (2004). Active living and Injury Risk. International Journal of Sports Medicine, 25(3), 209-216. https://doi.org/10.1055/s-2004-819935

Pierantozzi, E., \& Muroni, R. (2009). Judo high level competitions injuries. Medit J Musc Surv, 17, 26-9.

Pocecco, E., Ruedl, G., Stankovic, N., Sterkowicz, S., Del Vecchio, F., Gutiérrez-García, C., . . . Burtscher, M. (2013). Injuries in judo: a systematic literature review including suggestions for prevention. British journal of sports medicine, 47(18), 1139-1143. https://doi.org/10.1136/bjsports-2013-092886

Powell, J., \& Barber-Foss, K. (1999a). Injury patterns in selected high school sports: a review of the 1995-1997 seasons. Journal of Athletic Training, 34, 277-284.

Rechel, J., Yard, E., \& Comstock, D. (2008). An epidemiology comparasion of high school sports injuries sustained in practice and competition. Journal of Athletic Training, 43, 197-204. https://doi.org/10.4085/1062-6050-43.2.197

Saragiotto, B. T., Di Pierro, C., \& Lopes, A. D. (2014). Risk factors and injury prevention in elite athletes: a descriptive study of the opinions of physical therapists, doctors and trainers. Brazilian journal of physical therapy, 18(2), 137-143. https://doi.org/10.1590/S1413-35552012005000147

Shadgan, B., Feldman, B., \& Jafari, S. (2010). Wrestling injuries during the 2008 Beijing Olympic Games. The American Journal of Sport Medicine, 38(9), 1870-1876. https://doi.org/10.1177/0363546510369291

Sous, J., Ruiz, J., Brito, M., Navarro, R., Navarro, M.NR. (2006). Valoración de la incidencia de lesiones deportivas en luchadores canarios de alto nivel. 20 Jornadas canarias de traumatologia y cirugía ortopédica.

United Nations Educational, Scientific and Cultural Organization [UNESCO]. (2006). Collective consultation in view of proposing an International.

World Medical Association. (2013). World Medical Association Declaration of Helsinki: ethical principles for medical research involving human subjects. JAMA, 310, 2191-2194. https://doi.org/10.1001/jama.2013.281053

Yard, E., Collins, C., Dick, R., \& Comstock, R. (2008). An epidemiologic comparison of high school and college wrestling injuries. The American Journal of Sports Medicine, 36, 57-64. https://doi.org/10.1177/0363546507307507

Zetaruk, M., Violán, M., Zurakowski, D., \& Micheli, L. (2005). Injuries in martial arts: a comparison of five styles. British Journal of Sports Medicine, 39, 29-33. https://doi.org/10.1136/bjsm.2003.010322

Número de citas totales / Total references: $24 \underline{(100 \%)}$
Número de citas propias de la revista / Journal's own references: $0(0 \%)$

Rev.int.med.cienc.act.fís.deporte - vol. 18 - número 72 - ISSN: 1577-0354 